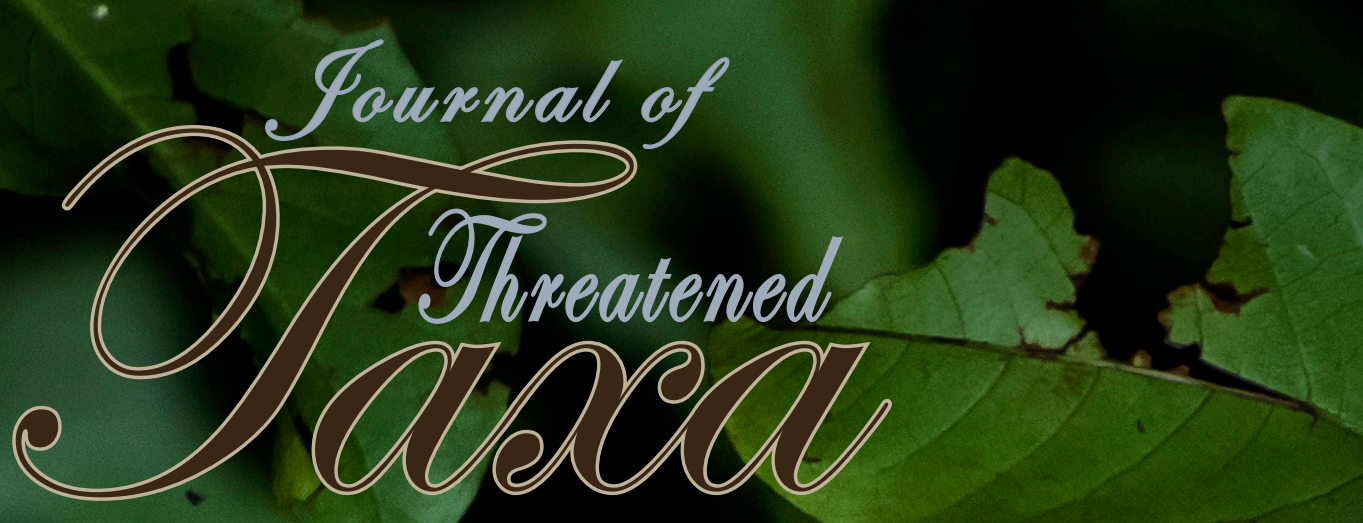

Building exidence for conservation glabally

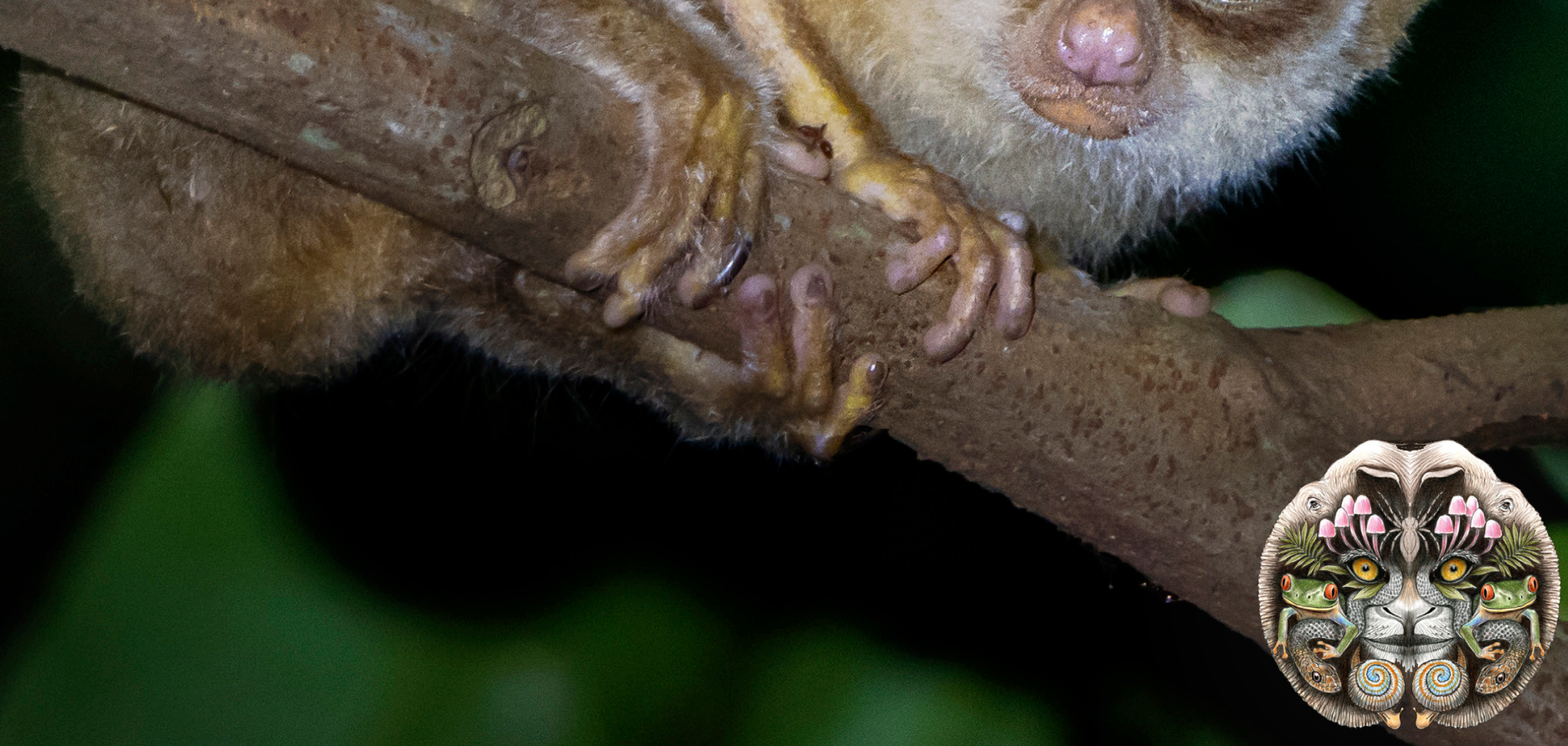

Open Access

$10.11609 /$ jott.2021.13.010.19431-19614 creven.threatenedtaxa.arg

26 September 2021 (Online \& Print) Val. 13 | Na. 11 | Pages: 19431-19674 


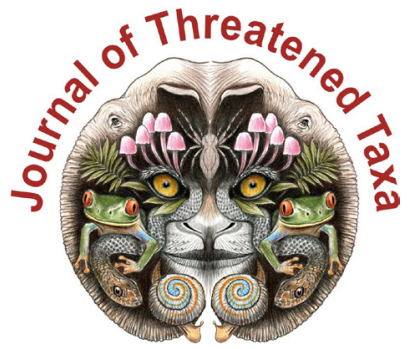

ISSN 0974-7907 (Online); ISSN $0974-7893$ (Print)

Publisher

Host

Wildlife Information Liaison Development Society

www.wild.zooreach.org

Zoo Outreach Organization www.zooreach.org

No. 12, Thiruvannamalai Nagar, Saravanampatti - Kalapatti Road, Saravanampatti, Coimbatore, Tamil Nadu 641035, India

Ph: +91 9385339863 | www.threatenedtaxa.org

Email: sanjay@threatenedtaxa.org

EDITORS

\section{Founder \& Chief Editor}

Dr. Sanjay Molur

Wildlife Information Liaison Development (WILD) Society \& Zoo Outreach Organization (ZOO)

12 Thiruvannamalai Nagar, Saravanampatti, Coimbatore, Tamil Nadu 641035, India

\section{Deputy Chief Edito}

Dr. Neelesh Dahanukar

Noida, Uttar Pradesh, India

\section{Managing Editor}

Mr. B. Ravichandran, WILD/ZOO, Coimbatore, India

\section{Associate Editors}

Dr. Mandar Paingankar, Government Science College Gadchiroli, Maharashtra 442605, India

Dr. Ulrike Streicher, Wildlife Veterinarian, Eugene, Oregon, USA

Ms. Priyanka Iyer, ZOO/WILD, Coimbatore, Tamil Nadu 641035, India

Dr. B. A. Daniel, $200 / \mathrm{WILD}$, Coimbatore, Tamil Nadu 641035, India

\section{Editorial Board}

Dr. Russel Mittermeier

Executive Vice Chair, Conservation International, Arlington, Virginia 22202, USA

\section{Prof. Mewa Singh Ph.D., FASc, FNA, FNASc, FNAPsy}

Ramanna Fellow and Life-Long Distinguished Professor, Biopsychology Laboratory, and Institute of Excellence, University of Mysore, Mysuru, Karnataka 570006, India; Honorary Professor, Jawaharlal Nehru Centre for Advanced Scientific Research, Bangalore; and Adjunct Professor, National Institute of Advanced Studies, Bangalore

\section{Stephen D. Nash}

Scientific Illustrator, Conservation International, Dept. of Anatomical Sciences, Health Sciences Center, T-8, Room 045, Stony Brook University, Stony Brook, NY 11794-8081, USA

\section{Dr. Fred Pluthero}

Toronto, Canada

\section{Dr. Priya Davidar}

Sigur Nature Trust, Chadapatti, Mavinhalla PO, Nilgiris, Tamil Nadu 643223, India

\section{Dr. Martin Fisher}

Senior Associate Professor, Battcock Centre for Experimental Astrophysics, Cavendish

Laboratory, JJ Thomson Avenue, Cambridge CB3 OHE, UK

\section{Dr. John Fellowe}

Honorary Assistant Professor, The Kadoorie Institute, 8/F, T.T. Tsui Building, The University of Hong Kong, Pokfulam Road, Hong Kong

\section{Prof. Dr. Mirco Solé}

Universidade Estadual de Santa Cruz, Departamento de Ciências Biológicas, Vice-coordenado do Programa de Pós-Graduação em Zoologia, Rodovia Ilhéus/Itabuna, Km 16 (45662-000)

Salobrinho, Ilhéus - Bahia - Brasil

\section{Dr. Rajeev Raghavan}

Professor of Taxonomy, Kerala University of Fisheries \& Ocean Studies, Kochi, Kerala, India

\section{English Editors}

Mrs. Mira Bhojwani, Pune, India

Dr. Fred Pluthero, Toronto, Canad

Mr. P. Ilangovan, Chennai, India

Web Maintenance

Mrs. Latha G. Ravikumar, ZOO/WILD, Coimbatore, India

\section{Typesetting}

Mr. Arul Jagadish, ZOO, Coimbatore, India

Mrs. Radhika, ZOO, Coimbatore, India

Mrs. Geetha, ZOO, Coimbatore India

\section{Fundraising/Communications}

Mrs. Payal B. Molur, Coimbatore, India

Subject Editors 2018-2020

Fungi

Dr. B. Shivaraju, Bengaluru, Karnataka, India

Dr. R.K. Verma, Tropical Forest Research Institute, Jabalpur, India

Dr. Vatsavaya S. Raju, Kakatiay University, Warangal, Andhra Pradesh, India

Dr. M. Krishnappa, Jnana Sahyadri, Kuvempu University, Shimoga, Karnataka, India

Dr. K.R. Sridhar, Mangalore University, Mangalagangotri, Mangalore, Karnataka, India

Dr. Gunjan Biswas, Vidyasagar University, Midnapore, West Bengal, India

Plants

Dr. G.P. Sinha, Botanical Survey of India, Allahabad, India

Dr. N.P. Balakrishnan, Ret. Joint Director, BSI, Coimbatore, India

Dr. Shonil Bhagwat, Open University and University of Oxford, UK

Prof. D.J. Bhat, Retd. Professor, Goa University, Goa, India

Dr. Ferdinando Boero, Università del Salento, Lecce, Italy

Dr. Dale R. Calder, Royal Ontaro Museum, Toronto, Ontario, Canada

Dr. Cleofas Cervancia, Univ. of Philippines Los Baños College Laguna, Philippines

Dr. F.B. Vincent Florens, University of Mauritius, Mauritius

Dr. Merlin Franco, Curtin University, Malaysia

Dr. V. Irudayaraj, St. Xavier's College, Palayamkottai, Tamil Nadu, India

Dr. B.S. Kholia, Botanical Survey of India, Gangtok, Sikkim, India

Dr. Pankaj Kumar, Kadoorie Farm and Botanic Garden Corporation, Hong Kong S.A.R., China

Dr. V. Sampath Kumar, Botanical Survey of India, Howrah, West Bengal, India

Dr. A.J. Solomon Raju, Andhra University, Visakhapatnam, India

Dr. Vijayasankar Raman, University of Mississippi, USA

Dr. B. Ravi Prasad Rao, Sri Krishnadevaraya University, Anantpur, India

Dr. K. Ravikumar, FRLHT, Bengaluru, Karnataka, India

Dr. Aparna Watve, Pune, Maharashtra, India

Dr. Qiang Liu, Xishuangbanna Tropical Botanical Garden, Yunnan, China

Dr. Noor Azhar Mohamed Shazili, Universiti Malaysia Terengganu, Kuala Terengganu, Malaysia Dr. M.K. Vasudeva Rao, Shiv Ranjani Housing Society, Pune, Maharashtra, India

Prof. A.J. Solomon Raju, Andhra University, Visakhapatnam, India

Dr. Mandar Datar, Agharkar Research Institute, Pune, Maharashtra, India

Dr. M.K. Janarthanam, Goa University, Goa, India

Dr. K. Karthigeyan, Botanical Survey of India, India

Dr. Errol Vela, University of Montpellier, Montpellier, France

Dr. P. Lakshminarasimhan, Botanical Survey of India, Howrah, India

Dr. Larry R. Noblick, Montgomery Botanical Center, Miami, USA

Dr. K. Haridasan, Pallavur, Palakkad District, Kerala, India

Dr. Analinda Manila-Fajard, University of the Philippines Los Banos, Laguna, Philippines

Dr. P.A. Sinu, Central University of Kerala, Kasaragod, Kerala, India

Dr. Afroz Alam, Banasthali Vidyapith (accredited A grade by NAAC), Rajasthan, India

Dr. K.P. Rajesh, Zamorin's Guruvayurappan College, GA College PO, Kozhikode, Kerala, India

Dr. David E. Boufford, Harvard University Herbaria, Cambridge, MA 02138-2020, USA

Dr. Ritesh Kumar Choudhary, Agharkar Research Institute, Pune, Maharashtra, India

Dr. Navendu Page, Wildlife Institute of India, Chandrabani, Dehradun, Uttarakhand, India

Invertebrates

Dr. R.K. Avasthi, Rohtak University, Haryana, India

Dr. D.B. Bastawade, Maharashtra, India

Dr. Partha Pratim Bhattacharjee, Tripura University, Suryamaninagar, India

Dr. Kailash Chandra, Zoological Survey of India, Jabalpur, Madhya Pradesh, India

Dr. Ansie Dippenaar-Schoeman, University of Pretoria, Queenswood, South Africa

Dr. Rory Dow, National Museum of natural History Naturalis, The Netherlands

Dr. Brian Fisher, California Academy of Sciences, USA

Dr. Richard Gallon, llandudno, North Wales, LL30 1UP

Dr. Hemant V. Ghate, Modern College, Pune, India

Dr. M. Monwar Hossain, Jahangirnagar University, Dhaka, Bangladesh

Mr. Jatishwor Singh Irungbam, Biology Centre CAS, Branišovská, Czech Republic

Dr. lan J. Kitching Natural History Museum, Cromwell Road, UK

Dr. George Mathew, Kerala Forest Research Institute, Peechi, India

Dr. John Noyes, Natural History Museum, London, UK

For Focus, Scope, Aims, and Policies, visit https://threatenedtaxa.org/index.php/JoTT/aims_scope
For Article Submission Guidelines, visit https://threatenedtaxa.org/index.php/JoTT/about/submissions
For Policies against Scientific Misconduct, visit https://threatenedtaxa.org/index.php/JoTT/policies_various 


\title{
A checklist of orthopteran fauna (Insecta: Orthoptera) with some new records in the cold arid region of Ladakh, India
}

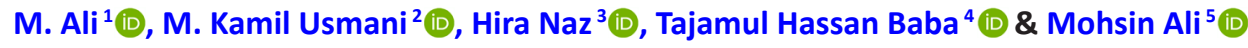 \\ 1,2,3,4 Department of Zoology, Aligarh Muslim University, Aligarh, Uttar Pradesh 202002, India. \\ ${ }^{5}$ Department of Zoology, Leh Campus, University of Ladakh, Uttar Pradesh 194101, India. \\ 19alimalla76@gmail.com (corresponding author), ${ }^{2}$ usmanikamil94@gmail.com, ${ }^{3}$ nazhiranaz@gmail.com, ${ }^{4}$ tajamul4u3@gmail.com, \\ ${ }^{5}$ mohsinzool82@gmail.com
}

\begin{abstract}
The study is mainly focused on the Orthopteran fauna of Ladakh. In the current field survey and literature survey, 29 species, 24 genera, 11 subfamilies, and five families belonging to four super families of Tettigonioidea (Krauss, 1902), Acridoidea (MacLeay, 1821), Eumastacoidea (Burr, 1899), and Pyrgomorphoidea (Burnner von Wattenwyl, 1847) are reported. The subfamily Gomphocerinae, and the following species Leva indica, Stenohippus mundus, Calliptamus italicus, Phaneroptera gracilis, Conocephalus longipennis, and C. maculatus are recorded for the first time from the region.
\end{abstract}

Keywords: Checklist, Orthoptera, new record, Ladakh.

The order Orthoptera comprises katydids, grasshoppers, locusts, and crickets. It is one of the largest insect orders having more than 28,000 species around the globe and over 1,200 species reported from India (Cigliano et al. 2020). Orthopteran fauna is widely distributed in all the ecological zones of the world but their distribution is dependent upon the vegetation like grasslands, forests, and agricultural fields. Some environmental factors like temperature, rainfall, and soil conditions also determine the distribution of grasshoppers. Orthopteran fauna play a significant role in the grassland ecosystem, they being important as primary consumers (herbivores) and also as contributors of diet to many other animals (reptiles, birds, amphibians, and mammals including man). Besides, Orthoptera plays a major role in the soil ecosystem by creating plant litter for soil, simultaneously plant growth and nutrients and cycling elements (Van Hook 1971).

Based on the size of the antennae, the order is divided into two suborders, Caelifera (short-horned) and Ensifera (long-horned). The suborder Ensifera is divided into seven superfamilies-Grylloidea, Gryllotalpoidea, Hagloidea, Stenopalmatoidea, Tettigonoidea, Rhaphidophoroidea, and Schazodactyloidea; whereas the suborder Caelifera into eight super families-Acridoidea, Eumastacoidea, Pneumoroidea, Proscopioidea, Pyrgomorphoidea, Tanoceroidea, Trigonopterygoidea, and Tetrigoidea. In Caelifera the superfamily Acridoidea shows the highest diversity with 11 families out of which the family Acrididae and Pyrgomorphidae are extensively distributed in India. Family Acrididae is divided into 27 subfamilies containing more than 800 genera which are also known as the most dominant and most diversified family in the order Orthoptera (Cigliano et al. 2020). A checklist of Indian Orthoptera including 1,033 species under 398 genera and 21 families was reported by Shishodia et al. (2010).

The remarkable taxonomic work on the Indian

Copyright: (c) Ali et al. 2021. Creative Commons Attribution 4.0 International License. JoTT allows unrestricted use, reproduction, and distribution of this article in any medium by providing adequate credit to the author(s) and the source of publication.

Funding: None.

Competing interests: The authors declare no competing interests.

Acknowledgements: The authors are highly thankful to the chairman, Department of Zoology, Aligarh Muslim University for lab facilities to carry out the research work. The first author is also thankful to UGC, New Delhi for providing the financial support. 
Acrididae was done by (Kirby 1914) in the book 'Fauna of British India' and divided the family into eight subfamilies. The checklist of Indian Acridoidea was firstly given by Tandon (1976). Bhomik (1984), Hazra et al. (1993), Tandon \& Shishodia (1995), Reshi et al. (2008), Sharma \& Mandal (2008), Sharma (2011), Rafi \& Usmani (2013), Rafi et al. (2014), and Kumar \& Usmani (2015) have contributed to the Indian Acrididae.

The present work was carried out to prepare a checklist of Orthoptera from the Ladakh region. The comprehensive study on Indian orthopteran fauna was published by Kirby (1914) and Chopard (1969). So, far there is no consolidated work on the orthopteran fauna of Ladakh is available; only some scattered information regarding orthopteran fauna of Ladakh have been published by a few researchers; Locust swarming at the two regions of Ladakh and major destruction caused by migratory locust Locusta migratoria migratoria in 2006 was studied by Ramamurthy \& Kumar (2009). The checklist of Jammu \& Kashmir (including Ladakh) has been prepared with 15 species from Ladakh by (Gupta \& Chandra 2018). Kumar et al. (2018) also reported 10 species of Orthoptera from Ladakh with some new records.

\section{MATERIALS AND METHOdS \\ Sampling site}

Ladakh: the region is located in the northern part of the country between $30.17 \mathrm{~N}$ latitude and $77.58 \mathrm{E}$ longitude having a total area $59,146 \mathrm{~km}^{2}$. The area is bounded in the north and east by China and in north-west by central Asia and Afghanistan (Figure 1). Geographically, Ladakh is the cradle inside the lofty Himalayan mountain ranges, which stretch south-east to north-east. A major part of it is inaccessible due to its high altitude which ranges from 2438 to 5486 meters above sea level. Most of the areas are infertile due to low rainfall, but those areas that are good in vegetation are where human habitation and water sources are available. Human settlement areas are richly vegetated due to irrigation. The area is commonly called 'cold desert' because it experiences both arctic and desert climate.

\section{Sample collection}

Adult specimens of both the sexes were collected from different areas comprising agricultural land, forest land, grassland, and rocky mountain areas by using the insect sweeping net and by handpicking method. The collections were made during the year 2018-2019 in the months of July, August, and September from various

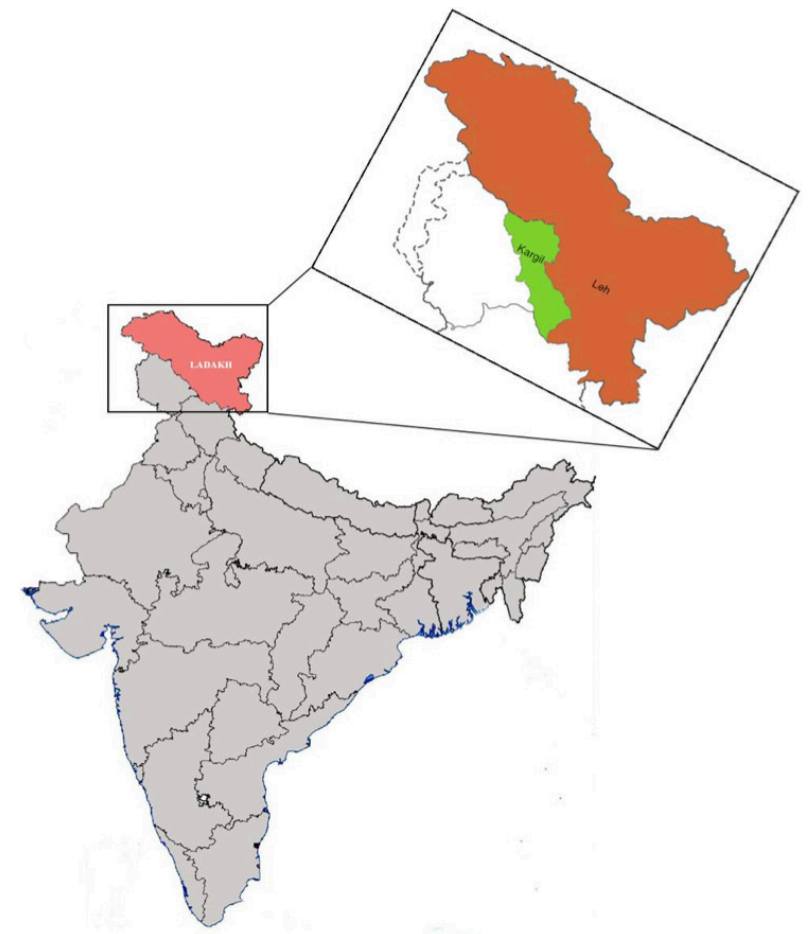

Figure 1. Map of Ladakh region.

places of Ladakh region.

An extensive literature survey was done to add the entire reported species from the region. All published information was undertaken by various sources which served as the basis for this critical analysis.

\section{Specimen preparation}

The specimens were killed by using ethyl acetate in an insect killing jar. After killing, the specimens were pinned and stretched with the help of the stretching board, the entomological pins used for specimen stretching and pinning were $0.3-0.4 \mathrm{~mm}$; the pins were inserted on the dorsum of pronotum slightly right to the median carina. The wings were stretched along with the right angle axis of the body, the hind legs slightly stretched backward along the axis of the body. The other body parts antennae, legs, and wings had to be supported with extra pins so that it could dry in the desired position. The specimens were removed from the stretching board after they were fully dried and stored in the insect collection box. Naphthalene balls were put in the corners of the collection box in order to prevent specimen deterioration.

\section{Species Identification}

After the collection, the adult specimens were studied under the binocular stereo zoom microscope 
and sorted out family-wise, sub-family-wise, generawise, and species-wise. The specimen identification was carried out with the help of key and description given by Bei-Bienko \& Mischienko (1964) and other keys available in the literature and on the website of the 'Orthopteran Species File'.

\section{RESULTS AND DISCUSSION}

During the recent survey, a total number of 29 species and 24 genera belonging to 11 subfamilies, and five families of Orthoptera were found to be represented from the Ladakh region. In the previous report of Jammu \& Kashmir, a total number of 15 species and 14 genera were recorded from the Ladakh region (Gupta \& Chandra 2018). Kumar et al. (2018) reported 10 species and six genera with two new records from the region. In the current study six species-Leva indica, Stenohippus mundus, Calliptamus italicus, Phaneroptera gracilis, Conocephalus longipennis, and Conocephalus sp.--are for the first time recorded from the region and the species Gyabus fusiformis rediscovered from the region (Image 1). A maximum number of species reported from the region belong to the subfamily Oedipodinae (8 genera, 12 species) followed by the subfamily Catantopinae and Gomphomastacinae (3 genera, 3 species), Calliptaminae and Gomphocerinae (2 genera, 2 species), Conocephalinae (1 genus, 2 species) and Conophyminae, Melanopolinae, Phenoropterinae, Pyrgomorphinae, and Tettigoniinae (1 genus, 1 species each) shown in Figure 2.

\section{Order Orthoptera Latreille 1793}

Suborder Caelifera Ander 1939

Superfamily Acridoidea Macleay, 1821

Family Acrididae Macleay, 1821

Subfamily Calliptaminae Jacobson, 1905

Genus Acorypha Krauss, 1877

1. Acorypha glaucopsis (Walker, 1870)

Caloptenus glaucopsis walker, F, 1870. Cata. Of the Specimen of Der. Salt. In Coll. Of British Museum 4:702.

Caloptenopsis glaucopsis Bolivar, I. 1917. Rev.real. Acad.Cienc.Exat. Fisic.Natur.16:409-410.

Acorypha glaucopsis Soomro, S. \& M.S. Wagan. 2005. Pakistan J. Zool. 37(3):230.

Acorypha glaucopsis Hemp, C. 2009. Journal of Orthopteran research 18(2):197.

Acorypha glaucopsis. Nayeem \& Usmani. 2012. Mun. Ento. \& Zoo. 7(1):409.

Acorypha glaucopsis Nazir, Mahmood, Ashfaq \& Rahim, 2014. JoTT 6(3):5544-5552.

Distribution: Somalia, Nigeria, Sudan, Iran, Yemen,
Tanzania, Pakistan, and

India (Madhya Pradesh, Karnataka, western Himalaya, Jammu \& Kashmir, Ladakh (Kargil), Tamil Nadu, Rajasthan, \& Himachal Pradesh).

Genus Calliptamus Serville, 183 I.

2. Calliptamus italicus (Linnaeus, 1758)

Gryllus (Locusta) italicus Linnaeus, 1758. Syst. Natur. Per Renga tria nature 1:432.

Gryllus italicus Thunberg, 1815. Mem. Acad. Imp. Sci.Sc. Peterburg 5:227 Calliptamus italicus. Lucas, P.H. 1851. Ann. Soc. ent. Fr. 9 2:363.

Caloptenus italicus Fischer, 1853. Ortho. Euro.377.

Caloptenus italicus Eversmann, 1859. Bull. Soc. Imp. Natur. Moscau 32(1): 138.

Calliptamus italicus Uvarov, 1922. Trans. R. Entomol. Soc. London. 48:136.

Calliptamus italicus Nagy, 2000. Duna. Dolg. Term. Tud. Sorozatt 10:155.

Calliptamus italicus italicus. Galvagni. 2010. Atti Acc. Rov. Agiati. 8 10(B):177.

Distribution: South-western Europe, Switzerland, Spain, France, Germany, Italy, Greece, Middle Europe, Africa, Turkey, Iran, Kazakhstan, Afghanistan, India (Jammu \& Kashmir and Ladakh), and China.

Subfamily Catantopinae Brunner and Wattenwyl, 1893 Genus Diabolocatantops Jago, 198

3. Diabolocatantops innotabilis (Walker, 1870)

Acridium innotabile Walker, F. 1870. Catalogue of the spec. of Dermap. In Collection of the British Museum 4:629.

Acridium innotabile Finot, 1907. Annal Society Ent. Fr. 76:336

Catantops innotabile Uvarov, 1929. Revue Suisse de Zool. 36:561.

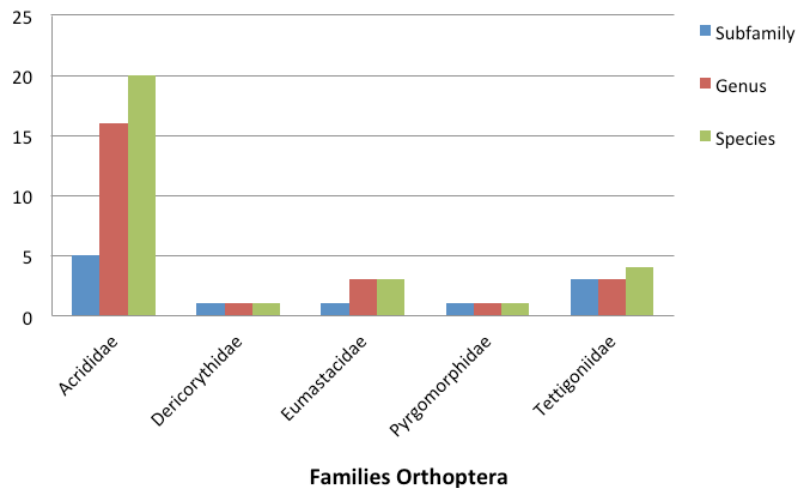

Figure 2. Showing the number of subfamilies, genera, and species of Orthoptera from Ladakh. 

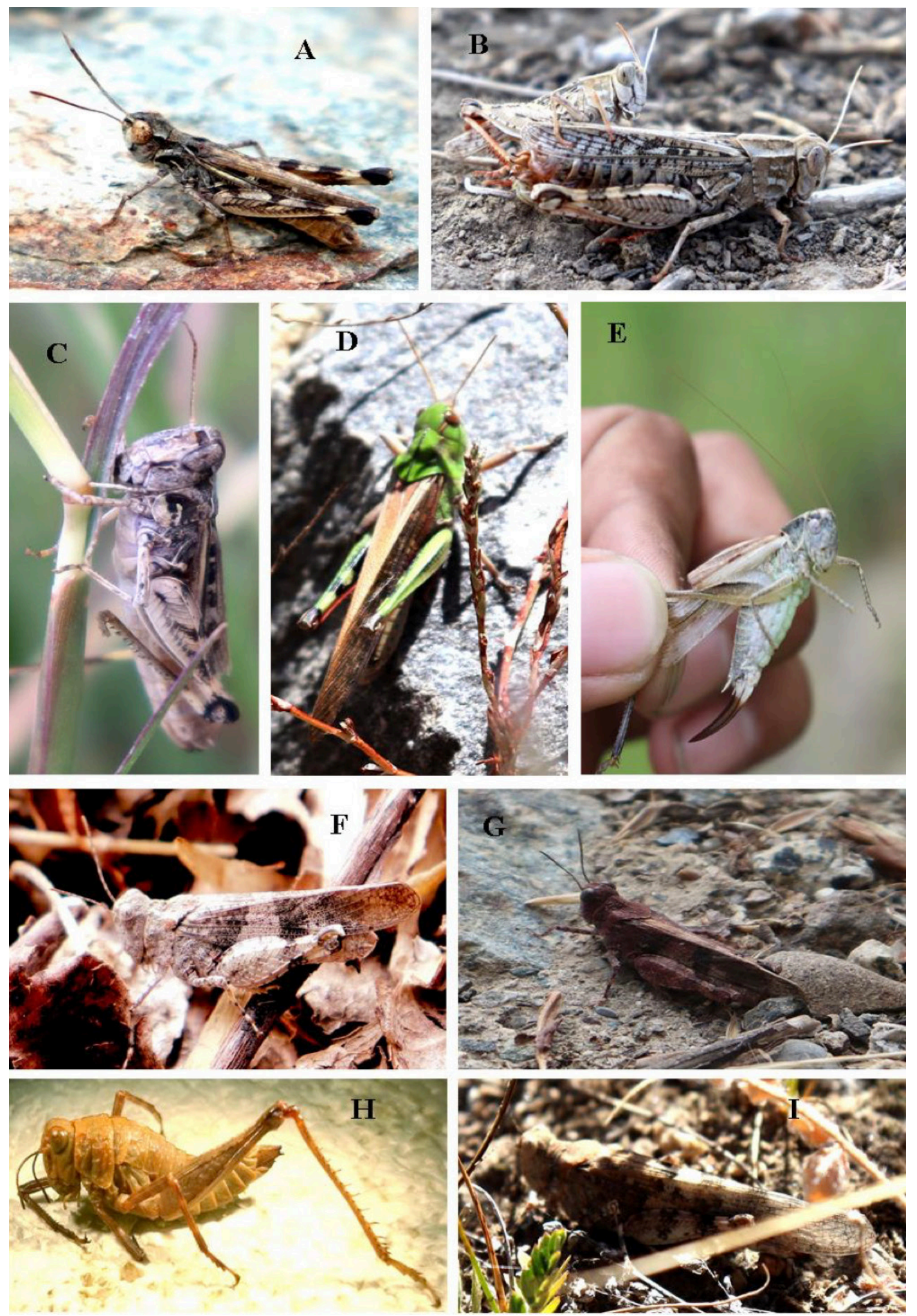

Image 1. Some collected specimens: A-Stenohippus mundus | B-Acorypha glaucopsis | C-Leva indica | D-Locusta migratoria migratoria | E-Conocephalus longipennis | F-Oedipoda miniata miniate | G-Oedipoda himalayana | H-Gyabus fusiformis | I-Sphingonotus savignyi. (c) Mohd Ali. 
Diabolocatantops innotabilis. Jago. 1984. Trans. Singapore.

Amer. Entomol. Soc. 110(3):371.

Diabolocatantops innotabilis Shishodia, Chandra and

Gupta, 2010. Rec. Zool. Sur. India Misc. Pub.314:39

Diabolocatantops innotabilis Kumar and Usmani, 2014. J. of Entomol. And Zool. Stud. 2(3):138

Distribution: Pakistan, India (Assam, Bihar, Jammu \& Kashmir, Ladakh: Leh (Nyoma), Maldives, Himachal Pradesh, Goa, Tamil Nadu, Nepal, Maharashtra. Uttarakhand, Uttar Pradesh and West Bengal.), Sri Lanka, Nepal and Thailand.

\section{Genus Paraconophyma Uvarov, 1921}

4. Paraconophyma scabra (Walker, 1870)

Caloptenus scaber Walker, F. 1870.Catalogue of the Specimens of Dermap. Salta.in the Collection of Brt.Mus. 4:707.

Mesambria scabra Kirby, W.F., 1910. A Synonymic Cat. of Orthop. 3(2):440. Paraconophyma scabra Uvarov, 1921. Ann. Mag. Nat. Hist. 97:501.

Paraconophyma scabra Bhomik, 1986. Zool. Surv. of India, Tech. Monogr.14:145.

Paraconophyma scabra Shishodia \& Tandon. 2004. Fauna of Manipur - Part 2.131.

Distribution: India (Bihar, Delhi, Himachal Pradesh, Jammu \& Kashmir, Ladakh: Leh (Nyoma Taklung) and West Bengal).

\section{Genus Xenocatantops Dirsh, 1953}

\section{Xenocatantops humilis humilis (Serville, 1838)}

Acridium humile Serville, 1838. Histoire naturelle des insectes. Orthopteres. 662.

Catantops humilis Karny, 1915.Supplementa Entomologica. 4:88

Catantops humilis. Uvarov. 1929. Revue Suisse de Zool. 36:561.

Xenocatantops hum ilis humilis Dirsh and Uvarov, 1953. Tijdschr. v. Entomologie 96:237

Xenocatantops humilis. Ingrisch. 1990. Spixiana (Munich). 13:175.

Xenocatantops humilis Cao \& Yin, 2007. Acta Zootaxonomica Sin 32(3):523

Xenocatantops humilis humilis Shishidia, Chandra and Gupta, 2010. Rec. Zool. Surv. India, Misc. Pub., Occas. Paper 314:37

Xenocatantops humilis. Tan, M.K. \& Kamaruddin. 2016. Zootaxa. 4111(1):26.

Distribution: India (Assam, Bihar, Himachal Pradesh, Uttarakhand, Jammu \& Kashmir, Ladakh: Leh (Nyoma), Mizoram, Sikkim, Tamil Nadu and West Bengal) Nepal, Bangladesh, Myanmar, Thailand., Malaysia and

Subfamily Gomphocerinae Fieber, 1853

Genus Leva Bolivar, 1909

6. Leva indica (Bolivar, 1902)

Gymnobothrus indicus Bolivar, 1902. Ann.So. ent. Fr. 70:596.

Leva indica Bolivar, 1902. Bol. R. soc.Esp. Hist. Nat. 9:292.

Leva indica Uvarov, 1929. Revue Suisse de Zool. 36:540.

Leva indica. Shishodia \& Tandon. 2000. Fauna of Tripura - Part 2.217.

Leva indica Nayeem and Usmani, 2012. Munis Ento. Zoo.7(1):410.

Distribution: India (Bihar, Manipur, Ladakh: Kargil, Tamil Nadu and Rajasthan) and Sri Lanka.

\section{Genus Stenohippus Uvarov, 1926}

7. Stenohippus mundus (Walker, 1871)

Stenobothrus mundus Walker, F., 1871. Catalogue of the Spec. of Derm. Salta.79.

Dociostaurus mundus Kirby, 1914. Fauna of Brit. India, Include. Ceylon and Burma. Orthoptera (Acrididae) 117, 119.

Stenohippus mundus Johnston, 1956. Annoat. Cata. of African Grasshoppers 689.

Leva (Stenohippus) mundus Jago, 1971. Proc. Acad. Nat. Sci. Philad. 123:223.

Leva mundus Bhowmik, 1990. Rec. Zool. Survey of India. 87(1-4):89-94.

Stenohippus mundus. Hodjat. 2015. J. Entomol. Res. Soc. $17(1): 98$.

Distribution: West tropical Africa, Burkina, Nigeria, Palestine, Iran, and India (Jammu and Kashmir, Ladakh: Kargil, Maharashtra, Mumbai and Rajasthan).

\section{Subfamily Melanopolinae Scudder, 1897}

Genus Dicranophyma Uvarov, 1921

\section{Dicranophyma babaulti Uvarov, 1925}

Dicranophyma babaulti. Uvarov. 1925. Mission Guy Babault dans. 1914. 1925:31, 33.

Dicranophyma babaulti Mani, M.S. 1968. Eco. And Bio. Of High Altitude Insects 212

Dicranophyma babaulti Shishodia, Chandra and Gupta, 2010. Rec. Zool. Sur. India, Misc. Publication, Occas. paper 314:79

Distribution: India (Jammu \& Kashmir, Ladakh: Kargil (Saliskote)). 
Subfamily: Oedipodinae MacLeay, 1871

Genus Ailopus Fieber, 1853

9. Ailopus simulatrix simulatrix (Walker, 1870)

Epacromia simulatrix Walker, F., 1870. Cata. of the Spec. of Dermap. Salta. In the collection of the British Museum 4:773.

Acrotylus simulatrix Kirby, 1910. A Synonymic Catalogue of Orthoptera 3(2):267.

Aiolopus simulatrix. Ingrisch. 1983. Nachrichtenbl. Bayer. Entom. 32(3):93.

Aiolopus simulatrix. Ingrisch. 1999. Esperiana. 7:361. Aiolopus simulatrix simulatrix. Usmani. 2008. Zootaxa. 1946:27.

Aiolopus simulatrix. Usmani. 2008. Insecta Mundi. 0041:10.

Aiolopus simulatrix simulatrix. Prabakar, Prabakaran \& Chezhian. 2015. Biolife. 3(1):348.

Distribution: Nigeria, Libya, Egypt, Turkey, Saudi Arabia, Yemen, Iran, Pakistan and India (Ladakh: Kargil (Saliskote), Maharashtra and Tamil Nadu).

\section{Genus Bryodema Fieber, 1853}

10. Bryodema luctuosum inda Saussure, 1884

Bryodema inda Saussure, 1884. Mem. Soc. Phys. Hist. Nat. Geneve. 28(9):181

Bryodema india Kirby, W.F. 1914. Fauna of British India, including Ceylon and Burma. Orthoptera (Acrididae) 151

Bryodema luctosum inda Bey-Bienko, 1930. Ann. Mus. ZoolAcad. Imp. Sciences St. Petersburg 31(1):116.

Bryodema luctuosum indum. Zhang, D.-C., Wenqiang Wang \& X. C. Yin. 2006. Entomol. News. 117(1):17.

Bryodema luctuosum indum Shishodia \& Gupta. 2009. JoTT. 1(11):569-572.

Distribution: India (Himachal Pradesh, Jammu \& Kashmir, Ladakh: Leh (Khardong La)) and China.

\section{Genus Gastrimargus Saussur, 1884}

11. Gastrimargus marmoratus (Thunberg, 1815) Gryllus marmoratus Thunberg, 1815. Mem. Acad. Imp. Science St. Peterburg 5:232.

Oedaleus (Gastrimargus)marmarotus Krauss, 1890. Zool. Jahr. Abt. Syst. Gergr. Und Biol. Der Tiere. 5(4):659. Oedaleus marmarotud Schulthess, 1898. Ann. Mus, Civ. Stor. Nat. Genova 39:187.

Gastrimargus marmoratus. Kirby, W.F. 1902. Trans. Entomol. Soc. Londo. 1902:71.

Gastrimargus marmoratus Willemse, C. 1930. Tijdschr. v. Entomo. 73:63.

Gastrimargus marmoratus Mahmood, K. Samira, Salmah \& Idris, 2008. Pakistan J. Zool.40(5):375.
Distribution: South Africa, India (Andhra Pradesh, Assam, Bihar, Sikkim, Jammu \& Kashmir, Ladakh (Nyoma), Uttarakhand, Uttar Pradesh and West Bengal) China, Myanmar, Malaysia, and Korea.

\section{Genus Locusta Linnaeus, 1758}

12. Locusta migratoria migratoria (Linnaeus, 1758)

Gryllus (Locusta) migratorius Linnaeus, 1758. Syst. Nat. pr Regna tria nature 1:432.

Gryllus migratorius Linnaeus, 1761. Fauna Sueciae sistens Animalia Sueciae 238.

Acrudium migratorium Lamarck, 1835. Hist. nat. Anim. Sans Vert. 4:444.

Oedipoda migratoria Selys Longchamps, 1850. Bull. Acad. Sci. Bruxelles 16(2):626-628.

Pachytylus migratoria Eversmann, 1859. Bull. Soc. imp. nat. Moscuau 32(1):139.

Pachytylus migratoria Dtein, J.P.E.F., 1878. Dtsch. Entomol.Z.22:233-236.

Pachytylus migratoria Schulthess, 1898. Ann. Mus. Civ. Stor. Nat. Genova 39:188.

Locusta migratoria Chopard, 1922. Faune de France 3:134, 161.

Locusta migratoria migratoria. Cejchan. 1963. Beitrage zur Entomologie. 13(7-8):781.

Locusta migratoria migratoria. LemonnierDarcemont, Puskás \& Darcemont. 2015. Articulata 30:63-80.

Distribution: India (Jammu and Kashmir, Himachal Pradesh, Ladakh: Kargil, Leh, Rajasthan and Uttar Pradesh) and All over the World.

\section{Genus Oedaleus Fieber, 1853}

13. Oedaleus abruptus (Thunberg, 1815)

Gryllus abruptus Thunberg, 1815. Mem. Acad. Imp. Sci. St. Peterburg 5:233.

Oedalueus abruptus Saussure, 1884. Mem. Soc. Phys. Hist. Nat. Geneve 28(9):110, 117.

Oedaleus abruptus Bolivar, I., 1917. Rev. Real Acad. Cienc. Exact., Fisic. Natur, 16:385.

Oedaleus abruptus Chang, K.S.F., 1939. Bull. Zool. Surv. India 6(1):20, 21.

Oedaleus abruptus Bhowmik \& Halder, 1984. Bull. Zool. Surv.India 6(1-3):48.

Oedaleus abruptus Lian, Y Hu \& Y Qiao. 2000. Entomotaxonomia. 22(3):171-174.

Oedaleus abruptus. Ingrisch. 2001. Senckenbergiana Biologica. 81:156.

Oedaleus abruptus Nayeem \& Usmani. 2012. Munis Entomology \& Zoology 7(1):408.

Distribution: Pakistan, India (Bihar, Delhi, Goa, 
Haryana, Jammu and Kashmir, and Ladakh: Indus River bank, Rajasthan, Manipur, Uttarakhand, Sikkim, Tripura, Tamil Nadu and West Bengal) Nepal, Thailand and Vietnam.

\section{Genus Oedipoda Latreille, 1829}

14. Oedipoda himalayana Uvarov, 1925

Oedipoda himalayana Uvarov, 1925. Mission Guy babaul dans, Acrididae 1925:22.

Oedipoda himalayana Bhomik, 1985. Rec. Zool. Surv. India, Mis. Pub., Occas. Paper 78:37.

Oedipoda himalayana Shishodia \& Gupta. 2009. JoTT 1(11):569-572.

Oedipoda himalayana. Azim, Reshi \& Rather. 2010. Halteres 1(2):8.

Distribution: India (Jammu \& Kashmir, Ladakh: Kargil, Himachal Pradesh and Uttarakhand) and Tibet.

\section{Oedipoda miniata miniata (Pallas, 1771)}

Gryllus miniatus Pallas, 1771. Reise durch Verschiedene Provinzen des Russ. Reiches 1:467.

Oedipoda miniata. Targioni-Tozzetti. 1891. Animali ed insetti del tobacco in erba e del tabacco secco. 152.

Oedipoda miniata Ebner, 1908. Verh. Der Zoologisch Botanischen Gesellsch. Wein 58:337.

Oedipoda miniata miniata Ebner, 1910. Zool. Jahr. Abt. Syst. Geogr. Und Biol. Der Tiere 1910: 401-414.

Oedipoda miniata Werner, 1938. S. B. Akad. Wiss. Wien, Math. Kl. 147:130.

Oedipoda miniata Johnston, H.B., 1956. Annoated catalogue of African grasshoppers 518.

Oedipoda miniata miniata Muraj, Dino \& Alimehilli, 1970. Bull. Univ. Shtet. Tiranes, Ser. Shken. Nat.24(3):139, 145.

Oedipoda miniata miniata Massa, Fontana, Buzzetti, Kleukers \& Ode 2012. Faunal d italia.orthoptera 48:434.

Oedipoda miniata miniata Defaut \& Morichon, 2015. Faune de france 97(1a,b):491.

Distribution: Europe, Libya, Turkey, Palestine, Russia, Iran, Kazakhstan, Pakistan and India (Jammu \& Kashmir, Ladakh: Kargil).

\section{Genus Sphingonotus Fieber, 1852}

16. Sphingonotus (Sphingonotus) eurasius eurasius Mischenko, 1937

Sphingonotus eurasius eurasius Mistshenko, 1937. Eos 12(3):193.

Sphingonotus eurasius Johnston, H.B., 1956. Ann. Cata. of African Grasshoppers 447.

Sphingonotus azurescens Harz, 1975.Ser. Entomol. 11:525,528.
Sphingonotus eurasius Badih \& F. Pascaul, 1998. Nouvelle Revue Ent. 15(2):134.

Sphingonotus eurasius Massa, 2009. Jour. Orth. Res. 18(1):84 .

Sphingonotus (Sphingonotus) eurasius eurasius Benediktov, 2009. Trudy Russk. Entomol. Obshch 80(1):24.

Sphingonotus eurasisus eurasius. Garai. 2010. Esperiana. 15:408. Sphingonotus (Sphingonotus) eurasius eurasius. Benediktov. 2011. Matériaux Orthopteriques et Entomocénotiques. 16:7.

Sphingonotus (Sphingonotus) eurasius eurasius Dey, L.S. Saboori, Hodjat, Tork, Pahlow \& Husemann, 2018. Zootaxa 4379(2):157.

Distribution: Morocco, Libya, Turkey, Palestine, Syria, Caucasus, Iran, Kazakhstan, India (Himachal Pradesh and Ladakh: Kargil (Hugnis)).

\section{Sphingonotus (Sphingonotus) rubescens fallax} Mishchenko, 1937

Sphingonotus fallax. Mistshenko. 1937(1936). Eos 12(3-4):153.

Sphingonotus rubescens fallax. Bey-Bienko \& Mistshenko. 1951. Locusts and Grasshoppers of the U.S.S.R. and Adjacent Countries. 2:620(269).

Sphingonotus rubescens fallax. Bhowmik. 1985. Rec. Zool. Surv. India, Misc. Pub., Occas. Paper. 78:41.

Sphingonotus (Sphingonotus) rubescens fallax. Shishodia, K. Chandra \& S.K. Gupta. 2010. Rec. Zool. Surv. India, Misc. Pub., Occas. Paper. 314:101.

Distribution: Europe, Africa, Afghanistan and India (Jammu \& Kashmir and Ladakh: Kargil, Leh).

\section{Sphingonotus (Sphingonotus) rubescens rubescens.} (Walker, 1870)

Oedipoda rubesens Walker, F., 1870. Zoologist 25(28):2301.

Sphingonotus rubescens Kirby, W.F., 1910. A Synonymic Catalogue of Orthoptera 3(2):274.

Sphingonotus rubescens Kirby, W,F., 1914. Fauna of British India, including Ceylon and Burma. Orthoptera (Acrdidae) 155.

Sphingonotus rubescens rubescens Mistshenko, 1937. Eos 12(3-4):169.

Sphingonotus (Sphingonotus) rubescens rubescens Dey, L.S., Saboori, Hodjat, Tork, Pahlow \& Husemann, 2018. Zootaxa 4379(2):167.

Distribution: Spain, Europe, Africa, Libya, Egypt, Turkey, Yemen, Palestine, Iran, Kazakhstan, Afghanistan and India (Jammu \& Kashmir and Ladakh: Kargil, Leh). 
19. Sphingonotus savignyi (Saussure, 1884)

Sphingonotus savignyi Saussure, 1884. Mem. Soc. Phys. Hist. Nat. Geneve 28(9):198.

Sphingonotus Savignyi Krauss, 1890. Verh. der Zool. Bota. Gesellsch. Wien. 28(9):198.

Sphingonotus savignyi Dirsh, 1965. The Afr. Gener. Of Acridoidea 470.

Sphingonotus savignyi savignyi Massa, 2009. Jour. Orth. Res. 18(1):470.

Sphingonotus(Sphingonotus) savignyi savignyi dey, L.S., Saboori, Hodjat, Tork, Pahlow \& Husemann. 2018. Zootaxa 4379(2):170.

Distribution: North Africa, Russia, Central Asia, Afghanistan, Pakistan and India (Jammu \& Kashmir, Ladakh: Kargil, Leh, and Himachal Pradesh).

\section{Genus Trilophidia Stal, 1873}

20. Trilophidia annulata (Thunberg, 1815).

Gryllus annulatus Thunberg, 1815, Mem. Acad. Imp.

Sci. St. Peterburg 5:234.

Trilophidia annulata Bolivar, I., 1902. Ann. Soc. Ent. Fr.70:604.

Trilophidia annulata Hollis, 1965. Trans. R. Entomol. Soc. London 117:251.

Trilophidia annulata Kumar and Usmani, 2016. Munis Entomology \& zoology 11(1): 83.

Distribution: Iran, Pakistan, India (Bihar, Jammu and Kashmir, Ladakh: Leh, Tamil Nadu, Maharashtra, Goa, Gujarat, Rajasthan, Orissa, Uttar Pradesh and West Bengal) Sri Lanka, Nepal, China, Thailand, Malaysia, Singapore, Korea and Japan.

Family Dericorythidae Jacobson \& Bianchi, 1905 Subfamily Conophyminae Mistshenko, 1952.

Genus Conophyma Zubovski, 1898.

21. Conophyma kashmiricum Mistshenko, 1950 Conophyma kashmiricum Mistshenko, 1950. C.R. Academic Science, URSS 72:213.

Conophyma kashmiricum Bey Bienko and Mistschenko, 1951. Locusta and Grasshoppers of the USSR and Adjacent countries 1:190(199).

Conophyma kashmiricum Balderson and Yin, 1991. Ento. Gaz. 42(3):195.

Distribution: India (Jammu \& Kashmir and Ladakh (Kargil-Matayen)).

Superfamily Eumastacoidea Burr, 1899

Family Eumastacidae Burr, 1899

Subfamily Gomphomastacinae Burr, 1899

Genus Gomphomastax Brunner Wattenwyl, 1898
22. Gomphomastax kashmirica Balderson \& Yin, 1991 Gomphomastax kashmirica Balderson \& Yin, 1991. Ento. Gazette. 42(3):191.

Gomphomastax kashmirica Usmani, Reshi \& Azim, 2008. Insecta Mundi 33:2

Distribution: India (Jammu \& Kashmir, Ladakh (TsoMorari)).

Genus Phytomastax Bey Bienko, 1949

23. Phytomastax bolivari (Uvarov, 1936)

Gomphomastax bolivari Uvarov, 1936.Opuscula Entomologica 1:18.

Phytomastax bolivari Bey Bienko \& Mistshenko, 1951. Locusta and Grasshoppers of the USSR and Adjacent Countries 1:122(128).

Gomphomastax bolivari Mani. 1968. Ecology and Biogeography of High Altitude Insects 212.

Phytomastrax bolivari Balderson \& Yin, 1991. Entomologist Gazette 42(3):192.

Distribution: India (Jammu \& Kashmir and Ladakh (Tragbal Pass)).

\section{Genus Gyabus Ozdikmen, 2008}

24. Gyabus fusiformis (Bei Bienko, 1949)

Pachymastax fusiformis Bey Bienko, 1949. C.R. Acad. Sci. URSS. 64(5):733.

Pachymastax fusiformis Bey Bienko, 1951. Locusta and Grasshoppers of the USSR and Adjacent Countries 1:118(126).

Gyabus fusiformis Ozdikmen, 2008. Zootaxa 1763:68. Distribution: India (Ladakh (Kargil-Choskor)).

Superfamily Pyrgomorphoidea Brunner Von Wattenwyl, 1874

Family Pyrgomorphidae Brunner Von Wattwnyl, 1874 Subfamily Pyrgomorphinae Burnner Von Wettenwyl, 1874

Genus Atractomorpha Saussure, 1872

25. Atractomorpha sinensis montana Kevan \& Chen, 1969

Actractomorpha sinensis montana Kevan, D,K,M, \& Y. K. Chen, Zoological Journal of Linnean Society 48:141. Atractomorpha sinensis montana Kevan, D.K.M., 1977. In Beier. Orthoperorum Catalogus 16:396.

Atractomorpha sinensis montana Vickery, 1996. Notes Lyman ent. Mus. Res. Lab 19:2-11.

Distribution: India (Jammu \& Kashmir and Ladakh).

Suborder Ensifera

Superfamily Tettigonioidea Krauss, 1902

Family Tettigoniidae Krauss, 1902 
Subfamily Conocephalinae Burmeister, 1838

Genus Conocephalus Thunberg, 1815

26. Conocephalus (Anisoptera) longipennis (Haan, 1843)

Locusta (Xiphidium) longipennis Haan, 1843. Temminck Verhandelingen over de Nederlansche Overzeesche Bezittingen 19/20:188,189.

Xiphidium longipenne Burnner von Wattenwyl, 1893. Ann. Mus. Civ. Stor. Nat. Genova213(33):181.

Conocephalus (Xiphidion) longipennis. Karny. 1912. Genera Insectorum. 135:11.

Conocephalus longipennis Pitkin, 1980. Bull. Br. Mus. (Nat. Hist) ent. 41(5):349.

Conocephalus (Anisoptera) longipennis Zhou, M., Bi \& Xian Wei Liu,2010.Zootaxa 2527:57.

Conocephalus (Anisoptera) longipennis . Kim, T.-W. \& Hong Thai Pham. 2014. Zootaxa 3811(1):69.

Conocephalus (Anisoptera) longipennis. Xiao, W., S.-

L. Mao, Jianfeng Wang \& J.H. Huang. 2016. Far Eastern Entomologist. 305:14.

Conocephalus (Anisoptera) longipennis. Nagar \&

Ranjni Swaminathan. 2016. Zootaxa. 4126(1):24.

Conocephalus (Anisoptera) longipennis. Farooqi \& Usmani. 2018. Zootaxa. 4461(3):390.

Distribution: Dakar, India (Andaman \& Nicobar, Assam, Karnataka, Kerala, Ladakh: Kargil and Uttar Pradesh), Eurasia, China, Malaysia, Vietnam, and Philippines.

27. Conocephalus (Anisoptera) maculatus (Le Guillou, 1841)

Xiphidion maculatus. Le Guillou. 1841. Revue et Magasin de Zoologie. 294.

Xiphidium (Xiphidium) maculatum Redtenbacher, 1891. Ver. der Zool. Bota. Gesellesch, Wein 41:515.

Anisoptera maculatum Kirby, W.F., 1906. A Synonymic Catalogue of Orthoptera (Orthoptera Saltatoria, Locustidae vel Acrididae) 2:278.

Conocephalus (Anisoptera) maculatus Hebard, 1992. Proc.Acad.Nat. Sci. Philad 74:243.

Conocephalus maculatus. Chopard. 1954. Mem. Inst. franc. Afr. Noire. 40(2):61.

Conocephalus (Anisoptera) maculatus Storozhenko, Kim \& Jeon, 2015. Monograph of Korean Orthoptera 45.

Conocephalus (Anisoptera) maculatus. Gaikwad, Koli, Raut, Waghmare \& Bhawane. 2016. JoTT. 8(2):8535.

Distribution: Africa, Libya, Saudi Arabia, Yemen, Pakistan, India (Orissa, Jammu and Kashmir, Ladakh: Kargil, Uttar Pradesh, Maharashtra and Uttarakhand) Nepal, China, Bhutan, Singapore, Malaysia, and Indonesia, Korea and Japan.
Subfamily: Phaneropterinae Burmeister, 1838.

Genus Phaneroptera Serville, 1831

28. Phaneroptera gracilis Burmeister, 1838

Phaneroptera gracilis Burmeister, 1838. Handbuch

der Entomologie 22(IVIII):690.

Phaneroptera subnotata. Burner von Wattenwyl.

1878. Monographie der Phaneropteriden. 2016.

Phaneroptera gracili. Karny, 1927. Zeitschr. Gesam. Naturwiss. 88:12.

Phaneroptera gracilis Ingrisch, 2002. Entomologica basiliensia. 24:124.

Phaneroptera gracilis Hugel, 2009. Zoosystema. 31(3):552.

Phaneroptera gracilis Shi, F.M., L.H. Zaho \& J.Jiao, 2013. Acta zootaxanomica Sin. 38(3):510.

Phaneroptera (Phaneroptera) gracilis gracilis Kim, T.W. \& Hong Thai Pham, 2014. Zootaxa. 38(3):510.

Distribution: South Africa, Pakistan, India (Ladakh, Uttar Pradesh, Eastern Himalaya and Tamil Nadu) Nepal, China, Bhutan and Malaysia.

\section{Subfamily Tettigoniinae Krauss, 1902}

Genus Hyphinomos Uvarov, 1921

29. Hyphinomos fasciata Uvarov, 1921.

Hyphinomos fasciata. Uvarov, 1921. Jour. Bombay Nat. Hist. Soc. 28:74.

Hypsinomus fasciata Mani, M.S., 1968,. Ecology and Biogeography of High Altitude Insects 212.

Hyphinomos fasciata. Gurney \& Liebermann. 1975. Jour. Wash. Acad. Sci. 65(3):102-107.

Distribution: Dakar, India (Jammu \& Kashmir and Ladakh: Kargil) and China.

\section{REFERENCES}

Bhowmik, H.K. (1984). Report on collection of Orthoptera (Insecta) from the district of Purulia and Bankura, West Bengal, India. Zoological survey of India 6(1-3): 109-114.

Chopard, L. (1969). The Fauna of India and the Adjacent Countries: Orthoptera, Vol. 2. Grylloidea. Zoological Survey of India, 415 pp.

Cigliano, M.M., H. Braun, D.C. Eades \& D. Otte (2020). Orthoptera Species File. Version 5.0/5.0. http://Orthoptera.SpeciesFile.org. Downloaded on 3 February 2020.

Gupta, S.K. \& K. Chandra (2018). An annotated check-list of Orthoptera (Insecta) from Jammu \& Kashmir, India. Munis Entomology and Zoology 13(2): 632-646.

Hazra, A.K., S.K. Tandon, M.S. Shishodia, A. Dey \& S.K. Mandal (1993). Insecta: Orthoptera: Acridoidea. In: Fauna of West Bengal, State Fauna Series 3(4): 287-354.

Kumar, H. \& M.K. Usmani (2015). A Review of the genus Hieroglyphus (Acrididae: Hemiacridinae) from India, with description of a new species. Tropical Zoology 28(2): 35-55.

Kirby, W.F. (1914). The fauna of British India including Ceylon and Burma, Orthoptera (Acrididae). London, ix+27pp.

Kumar, H. K. Chandra \& M. Ali (2018). 'On a collection of Acridoidea (Orthoptera) from Ladakh region of Jammu and Kashmir, India. Records of the Zoological Survey of India 118(4): 381-388. 
Rafi, U. \& M.K. Usmani (2013). Diversity and Distribution of Acridid Pests (Orthoptera: Acrididae) of Purvanchal region, Uttar Pradesh, India. Journal of the Bombay Natural History Society 110(1): 50-56.

Rafi, U., M.K. Usmani, M.H. Akhtar \& M.R. Nayeem (2014). Population Density, Diversity and Distributional Pattern of Grasshopper fauna (Acrididae: Acridoidea: Orthoptera) in central and eastern Uttar Pradesh, India. Records of the Zoological Survey of India 114(1): 165-176.

Reshi S.A., M.N. Azim \& M.K. Usmani (2008). A checklist of short horned grasshoppers (Orthoptera: Acridoidea) from Kashmir, India. Biosystematica 2: 25-32.

Sharma, N. \& S.K. Mandal (2008). Acridoidea Diversity of Hastinapur Wildlife Sanctuary, Uttar Pradesh, India. Record Zoological survey of India 108(3): 85-96.
Sharma, N. \& S.K. Mandal (2008). Acridoidea Diversity of Hastinapur Wildlife Sanctuary, Uttar Pradesh, India. Record Zoological survey of India 108(3): 85-96.

Sharma, N. (2011). Acridoidea (Orthoptera: Insecta) Diversity of Sur Sarovar Bird Sanctuary, Keetham, Agra (Uttar Pradesh, India). Record Zoological survey of India 111(2): 23-28.

Shishodia, M.S., K. Chandra \& S.K. Gupta (2010). An annotated checklist of Orthoptera (Insecta) from India. Record Zoological survey of India. 314:1-366.

Tandon, S.K. \& M.S. Shishodia (1995). Fauna of Western Himalaya, Orthoptera. Zoological Survey of India. Himalayan Ecosystem Series 1: $37-42$.

van Hook, R.I. (1971). Energy and Nutrient of Spider and Orthopteran Population in a Grassland ecosystem. Ecological Monographs 41: $1-26$.

Wilio 
Dr. Albert G. Orr, Griffith University, Nathan, Australia

Dr. Sameer Padhye, Katholieke Universiteit Leuven, Belgium

Dr. Nancy van der Poorten, Toronto, Canada

Dr. Kareen Schnabel, NIWA, Wellington, New Zealand

Dr. R.M. Sharma, (Retd.) Scientist, Zoological Survey of India, Pune, India

Dr. Manju Siliwal, WILD, Coimbatore, Tamil Nadu, India

Dr. G.P. Sinha, Botanical Survey of India, Allahabad, India

Dr. K.A. Subramanian, Zoological Survey of India, New Alipore, Kolkata, India

Dr. P.M. Sureshan, Zoological Survey of India, Kozhikode, Kerala, India

Dr. R. Varatharajan, Manipur University, Imphal, Manipur, India

Dr. Eduard Vives, Museu de Ciències Naturals de Barcelona, Terrassa, Spain

Dr. James Young, Hong Kong Lepidopterists' Society, Hong Kong

Dr. R. Sundararaj, Institute of Wood Science \& Technology, Bengaluru, India

Dr. M. Nithyanandan, Environmental Department, La Ala Al Kuwait Real Estate. Co. K.S.C., Kuwait

Dr. Himender Bharti, Punjabi University, Punjab, India

Mr. Purnendu Roy, London, UK

Dr. Saito Motoki, The Butterfly Society of Japan, Tokyo, Japan

Dr. Sanjay Sondhi, TITLI TRUST, Kalpavriksh, Dehradun, India

Dr. Nguyen Thi Phuong Lien, Vietnam Academy of Science and Technology, Hanoi, Vietnam

Dr. Nitin Kulkarni, Tropical Research Institute, Jabalpur, India

Dr. Robin Wen Jiang Ngiam, National Parks Board, Singapore

Dr. Lional Monod, Natural History Museum of Geneva, Genève, Switzerland.

Dr. Asheesh Shivam, Nehru Gram Bharti University, Allahabad, India

Dr. Rosana Moreira da Rocha, Universidade Federal do Paraná, Curitiba, Brasil

Dr. Kurt R. Arnold, North Dakota State University, Saxony, Germany

Dr. James M. Carpenter, American Museum of Natural History, New York, USA

Dr. David M. Claborn, Missouri State University, Springfield, USA

Dr. Kareen Schnabel, Marine Biologist, Wellington, New Zealand

Dr. Amazonas Chagas Júnior, Universidade Federal de Mato Grosso, Cuiabá, Brasil

Mr. Monsoon Jyoti Gogoi, Assam University, Silchar, Assam, India

Dr. Heo Chong Chin, Universiti Teknologi MARA (UiTM), Selangor, Malaysia

Dr. R.J. Shiel, University of Adelaide, SA 5005, Australia

Dr. Siddharth Kulkarni, The George Washington University, Washington, USA

Dr. Priyadarsanan Dharma Rajan, ATREE, Bengaluru, India

Dr. Phil Alderslade, CSIRO Marine And Atmospheric Research, Hobart, Australia

Dr. John E.N. Veron, Coral Reef Research, Townsville, Australia

Dr. Daniel Whitmore, State Museum of Natural History Stuttgart, Rosenstein, Germany.

Dr. Yu-Feng Hsu, National Taiwan Normal University, Taipei City, Taiwan

Dr. Keith V. Wolfe, Antioch, California, USA

Dr. Siddharth Kulkarni, The Hormiga Lab, The George Washington University, Washington,

D.C., USA

Dr. Tomas Ditrich, Faculty of Education, University of South Bohemia in Ceske

Budejovice, Czech Republic

Dr. Mihaly Foldvari, Natural History Museum, University of Oslo, Norway

Dr. V.P. Uniyal, Wildlife Institute of India, Dehradun, Uttarakhand 248001, India

Dr. John T.D. Caleb, Zoological Survey of India, Kolkata, West Bengal, India

Dr. Priyadarsanan Dharma Rajan, Ashoka Trust for Research in Ecology and the Environment (ATREE), Royal Enclave, Bangalore, Karnataka, India

\section{Fishes}

Dr. Neelesh Dahanukar, IISER, Pune, Maharashtra, India

Dr. Topiltzin Contreras MacBeath, Universidad Autónoma del estado de Morelos, México

Dr. Heok Hee Ng, National University of Singapore, Science Drive, Singapore

Dr. Rajeev Raghavan, St. Albert's College, Kochi, Kerala, India

Dr. Robert D. Sluka, Chiltern Gateway Project, A Rocha UK, Southall, Middlesex, UK

Dr. E. Vivekanandan, Central Marine Fisheries Research Institute, Chennai, India

Dr. Davor Zanella, University of Zagreb, Zagreb, Croatia

Dr. A. Biju Kumar, University of Kerala, Thiruvananthapuram, Kerala, India

Dr. Akhilesh K.V., ICAR-Central Marine Fisheries Research Institute, Mumbai Research

Centre, Mumbai, Maharashtra, India

Dr. J.A. Johnson, Wildlife Institute of India, Dehradun, Uttarakhand, India

\section{Amphibians}

Dr. Sushil K. Dutta, Indian Institute of Science, Bengaluru, Karnataka, India

Dr. Annemarie Ohler, Muséum national d'Histoire naturelle, Paris, France

\section{Reptiles}

Dr. Gernot Vogel, Heidelberg, Germany

Dr. Raju Vyas, Vadodara, Gujarat, India

Dr. Pritpal S. Soorae, Environment Agency, Abu Dubai, UAE.

Prof. Dr. Wayne J. Fuller, Near East University, Mersin, Turkey

Prof. Chandrashekher U. Rivonker, Goa University, Taleigao Plateau, Goa. India

Dr. S.R. Ganesh, Chennai Snake Park, Chennai, Tamil Nadu, India

Dr. Himansu Sekhar Das, Terrestrial \& Marine Biodiversity, Abu Dhabi, UAE
Birds

Dr. Hem Sagar Baral, Charles Sturt University, NSW Australia

Dr. Chris Bowden, Royal Society for the Protection of Birds, Sandy, UK

Dr. Priya Davidar, Pondicherry University, Kalapet, Puducherry, India

Dr. J.W. Duckworth, IUCN SSC, Bath, UK

Dr. Rajah Jayapal, SACON, Coimbatore, Tamil Nadu, India

Dr. Rajiv S. Kalsi, M.L.N. College, Yamuna Nagar, Haryana, India

Dr. V. Santharam, Rishi Valley Education Centre, Chittoor Dt., Andhra Pradesh, India

Dr. S. Balachandran, Bombay Natural History Society, Mumbai, India

Mr. J. Praveen, Bengaluru, India

Dr. C. Srinivasulu, Osmania University, Hyderabad, India

Dr. K.S. Gopi Sundar, International Crane Foundation, Baraboo, USA

Dr. Gombobaatar Sundev, Professor of Ornithology, Ulaanbaatar, Mongolia

Prof. Reuven Yosef, International Birding \& Research Centre, Eilat, Israel

Dr. Taej Mundkur, Wetlands International, Wageningen, The Netherlands

Dr. Carol Inskipp, Bishop Auckland Co., Durham, UK

Dr. Tim Inskipp, Bishop Auckland Co, Durham, UK

Dr. V. Gokula, National College, Tiruchirappalli, Tamil Nadu, India

Dr. Arkady Lelej, Russian Academy of Sciences, Vladivostok, Russia

Dr. Simon Dowell, Science Director, Chester Zoo, UK

Dr. Mário Gabriel Santiago dos Santos, Universidade de Trás-os-Montes e Alto Douro,

Quinta de Prados, Vila Real, Portugal

Dr. Grant Connette, Smithsonian Institution, Royal, VA, USA

Dr. M. Zafar-ul Islam, Prince Saud Al Faisal Wildlife Research Center, Taif, Saudi Arabia

Mammals

Dr. Giovanni Amori, CNR - Institute of Ecosystem Studies, Rome, Italy

Dr. Anwaruddin Chowdhury, Guwahati, India

Dr. David Mallon, Zoological Society of London, UK

Dr. Shomita Mukherjee, SACON, Coimbatore, Tamil Nadu, India

Dr. Angie Appel, Wild Cat Network, Germany

Dr. P.O. Nameer, Kerala Agricultural University, Thrissur, Kerala, India

Dr. Ian Redmond, UNEP Convention on Migratory Species, Lansdown, UK

Dr. Heidi S. Riddle, Riddle's Elephant and Wildlife Sanctuary, Arkansas, USA

Dr. Karin Schwartz, George Mason University, Fairfax, Virginia.

Dr. Lala A.K. Singh, Bhubaneswar, Orissa, India

Dr. Mewa Singh, Mysore University, Mysore, India

Dr. Paul Racey, University of Exeter, Devon, UK

Dr. Honnavalli N. Kumara, SACON, Anaikatty P.O., Coimbatore, Tamil Nadu, India

Dr. Nishith Dharaiya, HNG University, Patan, Gujarat, India

Dr. Spartaco Gippoliti, Socio Onorario Società Italiana per la Storia della Fauna "Giuseppe

Altobello", Rome, Italy

Dr. Justus Joshua, Green Future Foundation, Tiruchirapalli, Tamil Nadu, India

Dr. H. Raghuram, The American College, Madurai, Tamil Nadu, India

Dr. Paul Bates, Harison Institute, Kent, UK

Dr. Jim Sanderson, Small Wild Cat Conservation Foundation, Hartford, USA

Dr. Dan Challender, University of Kent, Canterbury, UK

Dr. David Mallon, Manchester Metropolitan University, Derbyshire, UK

Dr. Brian L. Cypher, California State University-Stanislaus, Bakersfield, CA

Dr. S.S. Talmale, Zoological Survey of India, Pune, Maharashtra, India

Prof. Karan Bahadur Shah, Budhanilakantha Municipality, Kathmandu, Nepal

Dr. Susan Cheyne, Borneo Nature Foundation International, Palangkaraja, Indonesia

Dr. Hemanta Kafley, Wildlife Sciences, Tarleton State University, Texas, USA

\section{Other Disciplines}

Dr. Aniruddha Belsare, Columbia MO 65203, USA (Veterinary)

Dr. Mandar S. Paingankar, University of Pune, Pune, Maharashtra, India (Molecular)

Dr. Jack Tordoff, Critical Ecosystem Partnership Fund, Arlington, USA (Communities)

Dr. Ulrike Streicher, University of Oregon, Eugene, USA (Veterinary)

Dr. Hari Balasubramanian, EcoAdvisors, Nova Scotia, Canada (Communities)

Dr. Rayanna Hellem Santos Bezerra, Universidade Federal de Sergipe, São Cristóvão, Brazil

Dr. Jamie R. Wood, Landcare Research, Canterbury, New Zealand

Dr. Wendy Collinson-Jonker, Endangered Wildlife Trust, Gauteng, South Africa

Dr. Rajeshkumar G. Jani, Anand Agricultural University, Anand, Gujarat, India

Dr. O.N. Tiwari, Senior Scientist, ICAR-Indian Agricultural Research Institute (IARI), New

Delhi, India

Dr. L.D. Singla, Guru Angad Dev Veterinary and Animal Sciences University, Ludhiana, India

Dr. Rupika S. Rajakaruna, University of Peradeniya, Peradeniya, Sri Lanka

Dr. Bahar Baviskar, Wild-CER, Nagpur, Maharashtra 440013, India

Reviewers 2018-2020

Due to pausity of space, the list of reviewers for 2018-2020 is available online.
The opinions expressed by the authors do not reflect the views of the Journal of Threatened Taxa, Wildlife Information Liaison Development Society, Zoo Outreach Organization, or any of the partners. The journal, the publisher, the host, and the partners are not responsible for the accuracy of the political boundaries shown in the maps by the authors.

\footnotetext{
Print copies of the Journal are available at cost. Write to:

The Managing Editor, JoTT,

c/o Wildlife Information Liaison Development Society,

No. 12, Thiruvannamalai Nagar, Saravanampatti - Kalapatti Road,

Saravanampatti, Coimbatore, Tamil Nadu 641035, India

ravi@threatenedtaxa.org
} 


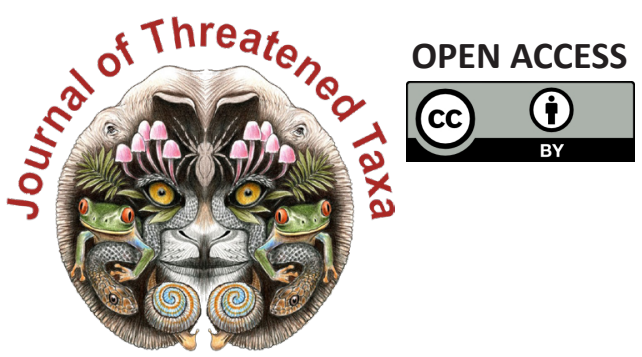

www.threatenedtaxa.org

The Journal of Threatened Taxa (JoTT) is dedicated to building evidence for conservation globally by publishing peer-reviewed articles online every month at a reasonably rapid rate at www.threatenedtaxa.org. All articles published in JoTT are registered under Creative Commons Attribution 4.0 International License unless otherwise mentioned. JoTT allows allows unrestricted use, reproduction, and distribution of articles in any medium by providing adequate credit to the author(s) and the source of publication.

\section{ISSN $0974-7907$ (Online) | ISSN $0974-7893$ (Print)}

\section{September 2021 | Vol. 13 | No. 11 | Pages: 19431-19674 Date of Publication: 26 September 2021 (Online \& Print) DOI: 10.11609/jott.2021.13.11.19431-19674}

Articles

Understanding human-flying fox interactions in the Agusan Marsh Wildlife Sanctuary as basis for conservation policy interventions

- Sherryl L. Paz \& Juan Carlos T. Gonzalez, Pp. 19431-19447

Argentinian odonates (dragonflies and damselflies): current and future distribution and discussion of their conservation

- A. Nava-Bolaños, D.E. Vrech, A.V. Peretti \& A. Córdoba-Aguilar, Pp. 19448-19465

\section{Communications}

The diel activity pattern of small carnivores of Western Ghats, India: a case study at Nelliampathies in Kerala, India

- Devika Sanghamithra \& P.O. Nameer, Pp. 19466-19474

Distribution and threats to Smooth-Coated Otters Lutrogale perspicillata (Mammalia: Carnivora: Mustelidae) in Shuklaphanta National Park, Nepal

- Gopi Krishna Joshi, Rajeev Joshi \& Bishow Poudel, Pp. 19475-19483

Wildlife hunting practices of the Santal and Oraon communities in Rajshahi, Bangladesh - Azizul Islam Barkat, Fahmida Tasnim Liza, Sumaiya Akter, Ashikur Rahman Shome \& M. Fazle Rabbe, Pp. 19484-19491

Ethnozoological use of primates in northeastern India

- Deborah Daolagupu, Nazimur Rahman Talukdar \& Parthankar Choudhury, Pp. 19492-19499

Factors influencing the flush response and flight initiation distance of three owl species in the Andaman Islands

- Shanmugavel Sureshmarimuthu, Santhanakrishnan Babu, Honnavalli Nagaraj Kumara \& Nagaraj Rajeshkumar, Pp. 19500-19508

Birds of Barandabhar Corridor Forest, Chitwan, Nepal

- Saneer Lamichhane, Babu Ram Lamichhane, Kapil Pokharel, Pramod Raj Regmi, Tulasi Prasad Dahal, Santosh Bhattarai, Chiranjibi Prasad Pokheral, Pabitra Gotame,

Trishna Rayamajhi, Ram Chandra Kandel \& Aashish Gurung, Pp. 19509-19526

On some additions to the amphibians of Gunung Inas Forest Reserve, Kedah,

Peninsular Malaysia

- Shahriza Shahrudin, Pp. 19527-19539

Reviews

A review of research on the distribution, ecology, behaviour, and conservation of the Slender Loris Loris lydekkerianus (Mammalia: Primates: Lorisidae) in India

- Mewa Singh, Mridula Singh, Honnavalli N. Kumara, Shanthala Kumar, Smitha D. Gnanaolivu \& Ramamoorthy Sasi, Pp. 19540-19552

Bivalves (Mollusca: Bivalvia) in Malaysian Borneo: status and threats

- Abdulla-Al-Asif, Hadi Hamli, Abu Hena Mustafa Kamal, Mohd Hanafi Idris, Geoffery James Gerusu, Johan Ismail \& Muyassar H. Abualreesh, Pp. 19553-19565

Disentangling earthworm taxonomic stumbling blocks using molecular markers

- Azhar Rashid Lone, Samrendra Singh Thakur, Nalini Tiwari, Olusola B. Sokefun \&

Shweta Yadav, Pp. 19566-19579

A reference of identification keys to plant-parasitic nematodes (Nematoda: Tylenchida) Tylenchomorpha)

- Reza Ghaderi, Manouchehr Hosseinvand \& Ali Eskandari, Pp. 19580-19602

Short Communications

Catalogue of herpetological specimens from Meghalaya, India at the Salim Ali Centre for Ornithology and Natural History

-S.R. Chandramouli, R.S. Naveen, S. Sureshmarimuthu, S. Babu, P.V. Karunakaran \&

Honnavalli N. Kumara, Pp. 19603-19610
A preliminary assessment of odonate diversity along the river Tirthan, Great Himalayan National Park Conservation Area, India with reference to the impact of climate change - Amar Paul Singh, Kritish De, Virendra Prasad Uniyal \& Sambandam Sathyakumar, Pp. 19611-19615

A checklist of orthopteran fauna (Insecta: Orthoptera) with some new records in the cold arid region of Ladakh, India

- M. Ali, M. Kamil Usmani, Hira Naz, Tajamul Hassan Baba \& Mohsin Ali, Pp. 19616-19625

New distribution records of two Begonias to the flora of Bhutan

- Phub Gyeltshen \& Sherab Jamtsho, Pp. 19626-19631

Rediscovery of Aponogeton lakhonensis A. Camus (Aponogetonaceae): a long-lost aquatic plant of India

- Debolina Dey, Shrirang Ramchandra Yadav \& Nilakshee Devi, Pp. 19632-19635

Glyphochloa acuminata (Hack.) Clayton var. laevis (Poaceae): a new variety from central Western Ghats of Karnataka, India

- H.U. Abhijit \& Y.L. Krishnamurthy, Pp. 19636-19639

A cytomorphological investigation of three species of the genus Sonchus L. (Asterales: Asteraceae) from Punjab, India

- M.C. Sidhu \& Rai Singh, Pp. 19640-19644

Dryopteris lunanensis (Dryopteridaceae) - an addition to the pteridophytic diversity of India

- Chhandam Chanda, Christopher Roy Fraser-Jenkins \& Vineet Kumar Rawat, Pp. 1964519648

Notes

First record of Spotted Linsang Prionodon pardicolor (Mammalia: Carnivora:

Prionodontidae) with photographic evidence in Meghalaya, India

- Papori Khatonier \& Adrian Wansaindor Lyngdoh, Pp. 19649-19651

First record of the Eastern Cat Snake Boiga gocool (Gray, 1835) (Squamata: Colubridae) from Tripura, India

- Sumit Nath, Biswajit Singh, Chiranjib Debnath \& Joydeb Majumder, Pp. 19652-19656

First record of the genus Tibetanja (Lepidoptera: Eupterotidae: Janinae) from India - Alka Vaidya \& H. Sankararaman, Pp. 19657-19659

Austroborus cordillerae (Mollusca: Gastropoda) from central Argentina: a rare, little-known land snail

- Sandra Gordillo, Pp. 19660-19662

Intestinal coccidiosis (Apicomplexa: Eimeriidae) in a Himalayan Griffon Vulture Gyps himalayensis

- Vimalraj Padayatchiar Govindan, Parag Madhukar Dhakate \& Ayush Uniyal, Pp. 1966319664

Two new additions to the orchid flora of Assam, India

- Sanswrang Basumatary, Sanjib Baruah \& Lal Ji Singh, Pp. 19665-19670

Wildlife art and illustration - combining black and white ink drawings with colour: some experiments in Auroville, India

- M. Eric Ramanujam \& Joss Brooks, Pp. 19671-19674
Publisher \& Host

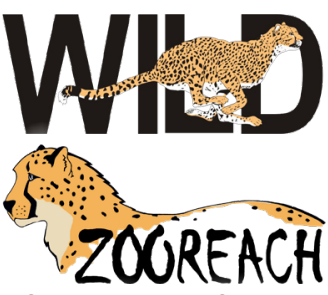

